\title{
Variability in content and dissolution profiles of MDMA tablets collected in the UK between 2001 and 2018 - a potential risk to users?
}

Couchman $L^{1,2}$, Frinculescu $A^{3}$, Sobreira $C^{3,4}$, Shine $T^{3}$, Ramsey ${ }^{3}$, Hecht $M^{1,5}$, Kipper $\mathrm{K}^{1,5}$, Holt DW ${ }^{1}$, Johnston $A^{1,4}$

${ }^{1}$ Analytical Services International, St. George's University of London, Cranmer Terrace, London SW17 ORE, UK.

${ }^{2}$ Pharmaceutical Sciences Clinical Academic Group, King's College London, London, UK.

${ }^{3}$ TICTAC Communications, St. George's University of London, Cranmer Terrace, London SW17 ORE, UK.

${ }^{4}$ Clinical Pharmacology, William Harvey Research Institute, Queen Mary University of London, London EC1M 6BQ, UK.

${ }^{5}$ Institute of Chemistry, University of Tartu, 14a Ravila Street, 50411 Tartu, Estonia.

\section{Declarations of interest:}

Part of this work was carried out using an instrument located at ASI for evaluation purposes, and has been acknowledged accordingly. MH worked at ASI during this study, and was supported by a Dora Plus scholarship fund, provided by the European Regional Development Fund and the Estonian government, and the European Regional Development Fund measure: 'Increasing the regional socio-economic impact of the RD\&I system and smart specialisation to develop growth areas'.

\section{Running head:}

Variability in dissolution profiles of MDMA tablets.

\section{Address for correspondence:}

Lewis Couchman

Analytical Services International (ASI), St. George's University of London Cranmer Terrace, London, SW17 ORE

Tel: +44 208725 2338, Fax: +442087679687

Email: lewis.couchman@bioanalytics.co.uk 


\section{ABSTRACT}

MDMA ('ecstasy') tablets are widely used recreationally, and not only vary in their appearance, but also in MDMA content. Recently, the prevalence of high-content tablets is of concern to public health authorities.

To compare UK data with other countries, we have evaluated MDMA content of 412 tablets collected from the UK, 2001-2018, and have investigated within-batch content variability for a sub-set of these samples. In addition, we have investigated dissolution profiles of tablets using pharmaceutical industry-standard dissolution experiments on 247 tablets. All analyses were carried out using LC-MS/MS.

Our data supported other studies, in that recent samples (2016 - 2018) tend to have higher MDMA content compared to earlier years. In 2018, the median MDMA content exceeded $100 \mathrm{mg}$ free-base for the first time. Dramatic within-batch content variability (up to $136 \mathrm{mg}$ difference) was also demonstrated. Statistical evaluation of dissolution profiles at 15-minutes allowed tablets to be categorised as fast-, intermediate-, or slowreleasing, but no tablet characteristics correlated with dissolution classification. Hence, there would be no way of users knowing a priori whether a tablet is more likely to be fast or slow-releasing. Further, within-batch variation in dissolution rate was observed.

Rapid assessment of MDMA content alone provides important data for harm reduction, but does not account for variability in (i) the remainder of tablets in a batch, nor (ii) MDMA dissolution profiles. Clinical manifestations of MDMA toxicity, especially for high-content, slow-releasing tablets, may be delayed or prolonged, and there is a significant risk of users re-dosing if absorption is delayed.

\section{KEYWORDS}

Ecstasy, MDMA, 3,4-methylenedioxymetamfetamine, dissolution, public health 


\section{INTRODUCTION}

3,4-Methylenedioxymetamfetamine (MDMA, 'ecstasy') is a common recreational drug, and is particularly popular amongst nightclub and music festival attendees due to the empathogenic/entactogenic nature of the MDMA 'high'1. Use is typically associated with younger populations. One study in the UK of 482 education centres found approximately one fifth (18.9\%) of the adolescents (16-21 years) questioned had at some time used $M M^{2}$. In England and Wales in 2017, there were 56 deaths attributed to MDMA toxicity ${ }^{3}$.

An additional consideration more recently is the availability of MDMA via the Darknet, purchased using cryptocurrencies, of which there are many ${ }^{4,5}$. There is some evidence that the perception amongst users is that drugs bought via the Darknet are (i) more likely to be 'as advertised', i.e. not containing an entirely different compound or not adulterated with other compounds and (ii) of higher content ${ }^{6-8}$. Though limited and not necessarily representative of the global marketplace, evidence available from the Netherlands suggests that MDMA tablets bought on-line are of a statistically significant, but only slightly higher content, than those bought on the street (131 and $121 \mathrm{mg}$, respectively) ${ }^{8}$. Current concerns for risks posed to recreational ecstasy users focusses on the prevalence of high-content MDMA tablets. Tablets containing 200-300 mg MDMA have been reported, including some tablets which had clearly been produced for sale at specific events, bearing the event logo'.

That users may be actively seeking means to ensure the MDMA content of the drugs they are buying is some evidence for the problem which exists regarding variability in drug dosage. Indeed, a number of 'home-testing' kits, typically using Marquis reagent or similar colour tests, are now commercially available ${ }^{10,11}$. Furthermore, variability in MDMA dosage (for tablets) and purity (for 'crystals') has previously been reported by a number of groups ${ }^{9,12-20}$, including a study previously carried out by this group for a sample of MDMA tablets collected in the UK in $2006^{21}$, which showed wide variability in the MDMA content of tablets in the UK [(range 20 - 131 mg MDMA hydrochloride per tablet (16.8 $110 \mathrm{mg}$ MDMA free-base)].

Dissolution testing is commonplace in the quality control and batch production of drugs in pharmaceutical laboratories, and for bioequivalence testing when developing new formulations or generic versions of existing drugs ${ }^{22,23}$. Standard protocols are available ${ }^{22}$. During drug and formulation development, dissolution data are used to determine pharmacokinetic parameters and guide decisions on dose frequency. Such data for illicit drugs are, unsurprisingly, sparse but are potentially of value for policy-makers and for 
informing harm reduction strategies. Notwithstanding the known problem of MDMA content variability, two MDMA tablets with markedly different dissolution profiles could pose significantly different clinical risks to users.

In this study, we have analysed MDMA tablets collected in the UK using liquid chromatography with tandem mass-spectrometry (LC-MS/MS). We have analysed representative samples from 2001 - 2018 to quantify MDMA content, including quantification for multiple tablets within a 'batch' to assess within-batch content variability. The study aims to update our previous study with more recent MDMA tablets, examine these data longitudinally, and to see how the UK data compare to those collected in Spain and Portugal ${ }^{24}$. In addition, we have taken a sub-set of MDMA tablets and, for the first time to our knowledge, report data from dissolution studies to demonstrate variability in MDMA tablet dissolution.

\section{METHODS}

\subsection{CHEMICALS AND REAGENTS}

MDMA and MDMA-D ${ }_{5}$ certified reference solutions $(1 \mathrm{~g} / \mathrm{L}$ and $100 \mathrm{mg} / \mathrm{L}$ in acetonitrile, respectively), MDMA hydrochloride reference standard, hydrochloric acid and formic acid were all from Sigma Aldrich (Poole, UK). LC-MS grade methanol was from Rathburn (Walkerburn, Scotland, UK). Isopropanol was from VWR (Lutterworth, UK). Water was deionised in-house (> 18 megohm cm, Elix ${ }^{\circledR}$, Watford, UK).

\subsection{MDMATABLETS AND CRYSTALS}

Tablets analysed were those collected from amnesty bins, or seized during entry searches, from a number of large music festivals and nightclubs in the UK. All were initially identified as potentially containing MDMA by visual identification, based on size, shape, colour and markings/logos, to tablets found to have contained MDMA previously. (TICTAC visual drug identification and information system for solid dose drugs, version 23.1, TICTAC Communications Ltd., London, UK) ${ }^{25}$. All TICTAC database entries were confirmed by gas chromatography mass-spectrometry (GC-MS) analysis (7890-5975C, Agilent Technologies, Santa Clara, CA). All tablets were stored in sealed $7 \mathrm{~mL}$ glass sample containers (VWR, Lutterworth, UK) at room temperature (nominally 20 to $25^{\circ} \mathrm{C}$ ). Only whole tablets were included in the analysis, i.e. any damaged tablets, or those with obvious fragments missing were excluded from the study.

For MDMA content measurement, a total of 412 tablets were analysed. Included were 249 single tablets from different sources, and 45 'batches' of tablets (i.e. multiple tablets 
from the same source, between 2 and 8 tablets per batch, total 163 tablets). The latter were used to assess within-batch variability in MDMA content. In addition, a subset of these samples ( $N=34,2004-2017$ ) were identified by TICTAC as containing a variety of additional, non-MDMA, compounds. For dissolution testing, 247 tablets (98 single tablets from different sources, and 61 batches of tablets - between 2 and 9 tablets per batch, as well as 6 crystal samples were analysed, 3 as 'bombs' wrapped in cigarette paper, and 3 as loose crystals). Although MDMA is usually present in 'street' samples as the hydrochloride salt, we express our findings as MDMA free-base as we did not determine which salt was present in the samples.

\subsection{MDMA CONTENT MEASUREMENT}

Tablets were crushed and homogenised using a clean agate pestle and mortar, and the crushed tablets were stored in glass sample containers at room temperature. For analysis, approximately $10 \mathrm{mg}$ of tablet was weighed accurately (AE240, Mettler Toledo, Leicester, UK), and the weighed powder transferred to a $100 \mathrm{~mL}$ volumetric flask, which was made to volume with a solution of $50 \%(\mathrm{v} / \mathrm{v})$ methanol in deionised water. After thorough mixing by inversion (30 min), $1 \mathrm{~mL}$ portions were diluted $(1+9, v / v)$ with $50 \%(v / v)$ methanol in deionised water and were stored refrigerated $\left(2-8^{\circ} \mathrm{C}\right)$ in $15 \mathrm{~mL}$ screw-cap tubes (Sarstedt, Leicester, UK) prior to analysis. MDMA calibrators ( $N=6,2 \mathrm{~mL}$ each calibration level) were prepared by dilution of MDMA stock solution ( $1 \mathrm{~g} / \mathrm{L})$ over the range $0.50-100 \mathrm{mg} / \mathrm{L}$ in $50 \%(\mathrm{v} / \mathrm{v})$ methanol in deionised water.

For analysis, prepared samples and calibrators $(50 \mu \mathrm{L})$ were diluted with $\mathrm{MDMA}_{5} \mathrm{D}_{5}$ solution (10 mg/L, $50 \mu \mathrm{L})$ in $1.5 \mathrm{~mL}$ micro-centrifuge tubes and $0.1 \%(v / v)$ formic acid in deionised water $(900 \mu \mathrm{L})$. Portions of these mixtures $(20 \mu \mathrm{L})$ were diluted further with $0.1 \%(v / v)$ formic acid in deionised water $(380 \mu \mathrm{L})$ directly into 96-well plates with eluent $A, 0.1 \%(v / v)$ formic acid in deionised water. Diluted samples $(2 \mu \mathrm{L})$ were injected onto a reversed-phase (C18), tapered-bore column $(2.7 \mu \mathrm{m}, 25 \times 2.0-0.5 \mathrm{~mm}$ i.d., WarpLCMS, Penn Valley, CA) and analysed using gradient elution (Table S1) on an Acquity $^{\mathrm{TM}}$ LC instrument (Waters, Manchester, UK). Analytes were monitored using a TQD MS (Waters, Manchester, UK), operating in positive electrospray ionisation, multiple reaction monitoring (MRM) mode. Ion transitions (collision energy, V) were: $m / z 194.1$ to 104.9 (24) and 163.0 (12), and 199.2 to 107.0 (26) and 165.0 (12) for MDMA and MDMA$D_{5}$, respectively. The dwell time for each MRM transition was $25 \mathrm{~ms}$. Ionisation source conditions were: capillary voltage $0.5 \mathrm{kV}$, cone voltage $3 \mathrm{~V}$, extractor voltage $3 \mathrm{~V}$, source temperature $150{ }^{\circ} \mathrm{C}$, desolvation gas temperature $450{ }^{\circ} \mathrm{C}$, and desolvation gas flow rate 900 L/h. Data were analysed using MassLynx ${ }^{\mathrm{TM}}$ (version 4.1) and Microsoft Excel (2016 version). 
For external quality control (EQC) to assess the method accuracy, portions of crushed MDMA tablets $(N=10)$ covering a range of MDMA content were sent to a second laboratory (LGC, Teddington, UK) for analysis. These were analysed by LGC without knowledge of the results obtained at ASI. The LGC results (MDMA content, as free-base, per tablet) were compared against those obtained by ASI. For internal quality control (IQC) and to assess between-assay precision, a selection of tablets ( $N=3$ of the 10 samples chosen for EQC) were chosen and were analysed with each of the batches.

For the tablets which were expected to contain additional compounds, extracted tablets were analysed using an LC-MS/MS method containing MRM transitions for $>100$ drugs and metabolites (2 MRM transitions per analyte, 12-minute gradient elution), used in our laboratory for routine forensic toxicology investigations.

\subsection{DISSOLUTION TESTING}

A 7-vessel dissolution bath (Pharma Test DT70, Hainburg, Germany) was used. Dissolution solvent ( $900 \mathrm{~mL}$ per vessel) was $0.05 \mathrm{~mol} / \mathrm{L}$ aqueous hydrochloric acid. Bath temperature was maintained at $37 \pm 0.5^{\circ} \mathrm{C}$, and the stirrer rate was $50 \mathrm{rpm}$. Samples $(5 \mathrm{~mL})$ were taken using a volumetric syringe via the sampling line and filter before addition of the tablet (t0), and subsequently after $5,10,15,20,30,45,60,90$, and 120 minutes. A final sample was drawn at 180 minutes (stirrer rate increased to $150 \mathrm{rpm}$ for the final 60 minutes). After each sample was drawn, the solution was replaced with $5 \mathrm{~mL}$ blank dissolution solution to maintain the vessel volume. Samples drawn at each time point were collected into 15 $\mathrm{mL}$ screw-cap tubes and stored at $2-8^{\circ} \mathrm{C}$ prior to analysis.

To confirm the stability of MDMA in the dissolution solution at for the 180-minute duration of the experiment, a solution of MDMA $(0.1 \mathrm{mg} / \mathrm{L}$, prepared in the dissolution solution) was incubated at $37^{\circ} \mathrm{C}$ and samples were drawn and analysed at each of the planned time points. This experiment also tested for any loss of analyte in the dissolution bath system (e.g. via adsorption to the vessel or syringe filter).

As for the content measurement, MDMA calibrators ( $\mathrm{N}=6,2 \mathrm{~mL}$ each calibration level) were prepared by dilution of certified MDMA reference solution $(1 \mathrm{~g} / \mathrm{L})$, this time over the range $0.50-200 \mathrm{mg} / \mathrm{L}$ in $50 \%(\mathrm{v} / \mathrm{v})$ methanol in deionised water. In addition, internal quality control (IQC) solutions were independently prepared from MDMA hydrochloride at $100,10.0$ and $1.00 \mathrm{mg} / \mathrm{L}(84.8,8.48$ and $0.85 \mathrm{mg} / \mathrm{L}$ MDMA free-base, respectively) in $50 \%(\mathrm{v} / \mathrm{v})$ methanol in deionised water.

For analysis of the collected samples, portions of the sample $(10 \mu \mathrm{L})$ were mixed with MDMA-D 5 solution $(20 \mu \mathrm{L}, 10 \mathrm{mg} / \mathrm{L})$ and diluted with $0.1 \%$ formic acid in deionised water 
$(1.5 \mathrm{~mL}$ ) directly into 96-well plates. Two injections were made from each well (one from each autosampler needle).

Analysis was carried out using a 1290 Infinity I $^{\text {TM }}$ LC system coupled with an Ultivo ${ }^{\mathrm{TM}}$ triple quadrupole mass-spectrometer (both Agilent Technologies, Santa Clara, CA, USA). Prepared samples $(2 \mu \mathrm{L})$ were injected using a dual-needle autosampler onto a Raptor ${ }^{\mathrm{TM}}$ biphenyl column (5.0 x $3.0 \mathrm{~mm}, 2.7 \mu \mathrm{m}$, Thames Restek, High Wycombe, UK) fitted directly to the MS ionisation source to permit rapid gradient analysis ${ }^{26}$. LC parameters are summarised in Table S1. Ionisation source conditions were: sheath gas temperature $350{ }^{\circ} \mathrm{C}$, sheath gas flow-rate $9 \mathrm{~L} / \mathrm{min}$, nebulizer $55 \mathrm{psi}$, drying gas temperature $350{ }^{\circ} \mathrm{C}$, drying gas flow-rate $11 \mathrm{~L} / \mathrm{min}$, capillary voltage $2.5 \mathrm{kV}$. Ion transitions (collision energy, $\mathrm{V}$ ) were: $m / z 194.1$ to 104.9 (17) and 163.0 (5), and 199.2 to 107.0 (21) and 165.0 (5) for MDMA and MDMA-D $D_{5}$, respectively. The dwell time for each MRM transition was $10 \mathrm{~ms}$, and the accelerator voltage was $9 \mathrm{~V}$.

\subsection{DATA PROCESSING}

Unlike pharmaceutical dissolution testing, in which all tablets are expected to contain the same dose of active pharmaceutical ingredient, the MDMA content of each tablet was unknown prior to analysis. Dissolution profiles were plotted as (i) concentration (mg/L) versus time and (ii) percent dissolved (relative to the 180 -minute time point) versus time. Statistical comparisons were made using One Way Analysis of Variance carried out using Minitab $^{\circledR}$ (version 18) and illustrated using Microsoft Excel (2016 version). For all calculations, MDMA was measured as free-base rather than the hydrochloride salt.

\section{RESULTS}

\subsection{MDMA CONTENT}

Calibration was linear over the range $0.50-100 \mathrm{mg} / \mathrm{L}$ (typical $\mathrm{r}^{2}>0.9984$ ). The withinbatch imprecision (\% CV) for the three IQC tablets (initial measured MDMA content 134, 71 and $34 \mathrm{mg}$ free-base) was $<13.3 \%$ for each of the five batches, and the between-batch imprecision was $<11.8 \%$. Comparison of the EQC sample results ( $\mathrm{N}=10$ tablets, MDMA content range $9-134 \mathrm{mg}$ by the in-house method) showed excellent agreement ( $y=$ $\left.1.0241 x-1.1456, r^{2}=0.9956\right)$. EQC results demonstrating our method accuracy are summarised in Figure S1. The stability of MDMA in the dissolution solution at $37{ }^{\circ} \mathrm{C}$ for the 180-minute duration of the experiment, was $98.0 \pm 1.5 \%$. 
For the tablets used to investigate MDMA content $(N=412)$, the median MDMA content tended to decrease overall between 2001 and 2009, but then increased from 2010 onwards (Figure 1). It should be noted that the number of tablets available for this study from 2009, 2010 and 2012 was low, 4, 2 and 6, respectively. This was attributed to the low purity and availability of MDMA-containing ecstasy during these years, and the increase in availability of then-legal novel psychoactive substances. That said, there was a statistically significant difference $(p<0.001)$ in MDMA content for those tablets collected pre-2010, and those collected post-2010 (Figure 2).

<<Figures 1-2 here >>

The median (range) MDMA content per tablet, tablet mass ( $\mathrm{mg}$ ) and MDMA content expressed as a percentage of the tablet mass by year is summarised in Table 1 . There was only a weak correlation between total tablet mass and MDMA content $\left(r^{2}=0.2919\right.$, Figure S2). Overall, the median tablet mass increased over the period studied (Figure 1).

$<<$ Table 1 here >>

The within-batch MDMA content and tablet mass variability ( $N=45$ batches of tablets) are summarised in Figure S3, ordered by year. The median (mean, range) difference in tablet mass (heaviest minus lightest tablet) within a batch was $5(14,0-80) \mathrm{mg}$. The median (mean, range) content difference (highest minus lowest MDMA content) was 10 $(19.5,2.2$ - 136) $\mathrm{mg}$. The mean within-batch precision in MDMA content (\% CV) was $16.4 \%$. Twenty two (49\%) of the batches had a within-batch precision less than $10 \%$.

Of the tablets tested which were expected to contain additional, non-MDMA, compounds, 22 (65\%) were found to contain caffeine. Other compounds identified (number of tablets) were: amfetamine (1), metamfetamine (1), 3,4-methylenedioxyethylamfetamine (MDEA) (3), cocaine (1), ketamine (3), methylephedrine (1), phentermine (1), 1-benzylpiperazine (BZP) (6), 1,4-dibenzylpiperazine (DBZP) (6), meta-chlorophenylpiperazine (mCPP) (3), diphenhydramine (2), and N,N-diallyl-5-methoxy-tryptamine (5-MeO-DALT) (1). From the visual identification made by TICTAC, two tablets were expected to contain methylone, in addition to MDMA, but were found to contain MDMA only. MDMA content of those with additional compounds was significantly lower than the MDMA-only tablets $(p<0.001)$.

\subsection{DISSOLUTION}

Calibration curves were fitted with a quadratic curve (weighted by the reciprocal of $x^{2}$, not forced through the origin) over the concentration range $0.1-200 \mathrm{mg} / \mathrm{L}$ (typical $\mathrm{r}^{2}>$ 0.9998). The within-batch and between-batch ( $N=9$ batches) precisions for the three 
independently-prepared IQC solutions were all $<13.7 \%$ and $<13.8 \%$, respectively. Mean within-batch accuracy (\% nominal concentration, $\mathrm{N}=30$ replicates of each IQC) was 92$96 \%$, and the mean between-batch accuracy ( $N=9$ batches) was $106-116 \%$.

The MDMA tablets were expected to be immediate release formulations and the 180-minute time point was chosen pragmatically as the time when dissolution was complete. Inspection of the dissolution curves showed plateaus of relative concentration confirming this expectation. Using data from the 180-minute time point, accounting for the dissolution vessel volume of $900 \mathrm{~mL}$, and assuming complete dissolution at the 180 minute time point, the MDMA content of each of the tablets included in the dissolution experiment was calculated.

Data from 247 dissolution experiments were included in the analysis. The median (25th and 75th percentiles) data from each time point were plotted to produce dissolution profiles, using both MDMA concentration $(\mathrm{mg} / \mathrm{L})$ versus time, and percent dissolved versus time. Assuming complete dissolution at 180 minutes, the concentration at this time point gave another measure of MDMA content and provided further data for the variability in MDMA content of the tablets tested; median (range) MDMA content $70 \mathrm{mg}$ (7 - $182 \mathrm{mg}$ ) free-base after accounting for dissolution vessel volume.

The distribution of the percent released was examined for each time point. Analysis of data at the 15-minute and 30-minute time points revealed a tri-modal distribution at 15 minutes, and a bi-modal distribution at 30 minutes. These were confirmed by analysis of variance $(p<0.001)$ which showed three distinct groups based on the percent dissolved at 15 minutes; mean (95\% Cl) percent dissolved: fast-releasing $95.0 \%$ (93.4 - $96.6 \%)$, intermediate-releasing $68.4 \%$ (66.5 - 70.3\%), and slow-releasing $39.4 \%$ (37.7 - $41.1 \%)$ Figure $3 \mathrm{~A}$ - and two distinct groups at 30 minutes: fast-releasing $95.7 \%$ (94.4- $97.1 \%$ ) and slow-releasing $57.3 \%$ (55.2 - 59.3\%) - Figure 3B. Using these distribution data, tablets were then classified for each of the two time points into (i) slow-releasing ( $\leq 54 \%$ dissolved), (ii) intermediate-releasing ( $55-81 \%$ dissolved), or (iii) fast-releasing ( $\geq 81 \%$ dissolved) for the 15-minute time point, and as (i) slow-releasing ( $<77 \%$ dissolved) or (ii) fast-releasing ( $\geq 77 \%$ dissolved) for the 30-minute time point. Dissolution profiles produced for each tablet class using the median (25th and 75th percentiles) percent dissolved at each time point are shown in Figure 4. At the 15-minute time point, there were 95 (38\%) fast-releasing tablets, 67 (27\%) intermediate-releasing tablets, and 85 (35\%) slow-releasing tablets. At the 30-minute time point, there were 172 (69\%) fastreleasing tablets and 75 (31\%) slow-releasing tablets. The highest content slow-releasing tablet (based on the 15-minute time point) contained $156 \mathrm{mg}$ MDMA free-base (185 mg 
MDMA $\mathrm{HCl}$ ). All MDMA crystal samples, including those wrapped in 'bombs', were classified as fast-releasing at both time points. There were no statistically significant differences in MDMA content based on dissolution classification at either 15-miute ( $p=$ 0.527 ) or 30-minute $(p=0.067)$ time points (Figure 5$)$. Furthermore, dissolution classification was not always consistent within the batch of tablets. At the 30-minute time point, there was discrepant classification (that is, at least one tablet in the batch was classified differently to the remainder) in 10 (16\%) of the batches tested (for example, Batch 89, $\mathrm{N}=5$ tablets, contained two fast-releasing and three slow-releasing tablets).

<<Figures 3-5 here>>

The proportion of fast-releasing, intermediate-releasing, and slow-releasing tablets by year is shown in Figure 6. Overall, the proportion of slow-releasing tablets decreased (64.3\% of tablets $2001-2003$ to $17.0 \%$ of tablets $2016-2018$ ), and the proportion of fast-releasing tablets increased from $21.4 \%$ of tablets $2001-2003$ to $52.3 \%$ of tablets 2016 - 2018 over the period studied, but there was still significant between-tablet variability in dissolution in each year group.

<<Figure 6 here»>

\subsection{TABLET CHARACTERISTICS - CORRELATION WITH MDMA CONTENT AND DISSOLUTION.}

With few exceptions (for example a batch of heart-shaped tablets in 2005), round/circular tablets were predominant until 2010. Thereafter, more elaborate-shaped tablets became more common. Likewise, prior to 2010 , the majority of tablets were white in colour, after which more colourful, and even multi-coloured tablets became more common (Figure 7). Statistically, white tablets and round tablets contained significantly less MDMA than the remainder of the tablets tested (both $p<0.001$ ). There was no association (Chi-squared test) between the white/non-white tablets and the dissolution classification at either 15minutes $(p=0.272)$ or 30 minutes $(p=0.729)$, nor between the round/non-round tablets and dissolution classification at either time point $(p=0.055$ and 0.246 at 15 -minutes and 30-minutes, respectively).

<<Figure 7 here >

\section{DISCUSSION}

Previous studies have highlighted the risk of variability in MDMA content of ecstasy tablets $^{9,27}$. Furthermore, a number of studies have suggested that in recent years, the 
amount of MDMA in tablets is increasing ${ }^{9}$. Data from the UK presented from the MDMA content measurement portion of this study support these observations (Figure 8 ) $^{24}$. Our data from 2006 showed the highest content tablet contained $110 \mathrm{mg}$ MDMA free-base (131 mg MDMA HCl) ${ }^{21}$. However, in 2018, the median MDMA content exceeded $100 \mathrm{mg}$ (equivalent to $118 \mathrm{mg}$ MDMA hydrochloride) for the first time, and the highest content tablet contained $241 \mathrm{mg}$ MDMA free-base (286 mg MDMA HCl), more than double the content of the highest of the 2006 tablets (Table 1). We observed a statistically significantly lower MDMA content for white and for round tablets, though this can be explained by the fact that these tablets tended to be from earlier years, when MDMA content was lower (Figure 1). That said, white, round tablets should not universally be considered 'low-dose' - one such tablet from 2001 had an MDMA content of $184 \mathrm{mg}$ freebase (219 mg MDMA HCl). Two tablets from a batch which were labelled with '199.9 mg' (presumably to reflect the apparent MDMA 'dose') were in fact found to contain 78.3 and 90.0 mg MDMA free-base (Figure 7).

<<Figure 8 here >>

The samples collected and used for this study provided the opportunity to investigate the variability in MDMA content within batches of tablets. Though for many of the batches tested the number of tablets was low (minimum 2 tablets per batch), the data revealed that the within-batch reproducibility of tablet mass was generally good. This is likely a reflection of the reproducibility of modern tablet-pressing equipment ${ }^{9}$. However, some batches showed large differences, even in the mass of tablets. One batch (Batch 14, N = 4) showed a difference of $80 \mathrm{mg}$ between the heaviest and lightest tablet in the batch (MDMA content of the heaviest and lightest tablets in this batch 46 and 22 mg MDMA free-base, respectively).

With respect to MDMA content of tablets within batches, there was greater variability than in the tablet mass (median difference in MDMA content $10 \mathrm{mg}$ ). Almost half the batches tested showed 'good' tablet-to-tablet reproducibility (\% CV $<10 \%$ ). However, a number of batches showed marked differences in MDMA content despite similar tablet mass. For example, in one batch (Batch $1, \mathrm{~N}=4$ tablets), despite all tablets being very similar in mass (range 256-259 mg), significant variability in content was observed (range 70-184 mg MDMA free-base). When producing tablets in the pharmaceutical industry, homogeneity of the bulk powder using high-shear mixing tools is essential to ensure content uniformity of the active pharmaceutical ingredient, and forms an integral part of release-testing protocols ${ }^{28}$. Variability in MDMA content within a batch of illicit tablets, when the tablet mass is consistent, most likely represents incomplete mixing of the MDMA and tablet excipients prior to pressing and of course demonstrates the inevitable 
lack of quality control for illicitly produced tablets. For MDMA users, our data suggest that analysis of a single tablet from a batch cannot guarantee that all others in the same batch are of the same content. For users tending to base their tablet ingestion (i.e. the number of tablets) on previous experiences, this could prove dangerous. For instance, a user may be 'accustomed' to taking $200 \mathrm{mg}$ MDMA per night. If they are informed upon analysis that a batch of tablets each contain $50 \mathrm{mg}$ MDMA, then they will plan to ingest four tablets from that batch. In reality, content variability of MDMA tablets may mean that, in total, they far exceed this dose, and hence increase their risk of toxicity.

LC-MS/MS was chosen as the analytical method for these studies for a number of reasons. Primarily, the specificity of mass-spectrometry when compared to the more normal UV detection allowed for the analysis of tablets with unknown excipients, contaminants, or adulterants which may interfere in UV systems. In addition, LC-MS/MS allowed for the inclusion of isotopically-labelled MDMA as an internal standard for quantitation. Finally, for the dissolution experiments in particular, where the number of samples to be analysed was large, ultra-rapid, high-throughput analysis was permitted (36 s per injection).

Measurement of MDMA content is useful for implementing harm-reduction strategies and providing information on trends for both MDMA users and healthcare professionals. Typically, as in the first part of this study, these data are presented as retrospective audits of substances collected or purchased over a number of years, carried out in laboratory settings by groups such as the Drug Information and Monitoring System (DIMS) in the Netherlands, which has been in existence since $1992^{29,30}$ and the National Detection System of Drugs and Toxic Substances (SINTES) in France ${ }^{13}$. Some laboratory-based groups, e.g. DanceSafe in the United States and Canada, and most recently the Welsh Emerging Drugs and Identification of Novel Substances (WEDINOS) Project in Wales and the UK publish their analytical findings online ${ }^{31-33}$. A review of the analytical techniques used in European testing laboratories was compiled as part of the Trans-European Drug Information (TEDI) project ${ }^{20,34}$.

Non-laboratory-based ('in-field' or mobile) testing of MDMA and other substances is also commonly carried out, for instance at music festivals, nightclubs, and in city-centres, with the aim of rapidly informing users of risk(s), e.g. due to circulation of high-content tablets, and in efforts to reduce harm. A number of organisations, including some already mentioned that also offer laboratory-based testing, carry out such testing services, e.g. Check It! (Austria), Check !n (Portugal), Energy Control (Spain), Safer Dance (Switzerland) and The Loop $(U K)^{30}$. Field-based drug identification tends to be limited to colourimetric reagent tests, spectroscopic methods, e.g. infra-red (IR), Fourier transform IR (FTIR) and Raman spectroscopy, and occasionally thin layer chromatography. Whilst these methods 
are cheap, rapid, and easily carried out without the need for elaborate laboratory infrastructure, colourimetric reagent tests are highly subjective, and there are limitations of spectroscopic methods in both sensitivity and specificity (particularly for tablets and powders containing mixtures of compounds or for very low concentration adulterants) ${ }^{20,35-37}$. Further, the results produced by spectroscopic methods are dependent on (i) up-to-date libraries to include the latest substances, and (ii) complex inbuilt identification algorithms for the analysis of mixtures. For the latter, such libraries are now available, and are ever-growing to include new compounds, e.g. TICTAC spectral libraries for novel psychoactive substances ${ }^{38}$. Mobile HPLC with ultraviolet and MS detection (single quadrupole) has also been used by the Check It! organisation in Austria, with throughput of approximately 40 samples per hour, using a modified laboratory van which can be deployed to music festivals. The LC-MS/MS method used for the dissolution profiling in this study provides additional evidence that, although significantly more expensive than colourimetric and spectroscopic methods, these specific and sensitive methods can in fact be carried out very rapidly (up to one 96-well plate per hour throughput using the method described). Through implementing novel, miniaturised instrumentation, such as the triple quadrupole MS used for the dissolution analyses in this study (Ultivo ${ }^{\mathrm{TM}}$ ), these techniques are also becoming more amenable to nonlaboratory environments.

Most importantly, the data in the second part of this study showed significant differences in the dissolution profile of MDMA tablets. These data highlight an extremely important additional risk with regards variability in MDMA tablets. There is little doubt that highercontent MDMA tablets pose a greater risk of toxicity to users than low-content tablets ${ }^{27,39,40}$. Content variability aside, risks for fast-releasing and slow-releasing tablets should also be considered, especially since our data suggest no definite way to know $a$ priori which class a tablet is likely to be in (e.g. based on colour or shape), and there were no statistically significant differences in MDMA content for fast-releasing, intermediatereleasing, or slow-releasing tablets. For high-content tablets, if the tablets are fastreleasing, toxicity may manifest rapidly (within 15 minutes or less) and hence will require rapid emergency treatment in cases of overdose. However, the highest risk could be posed by high-content, slow-releasing tablets. Peak plasma concentrations following oral administration of these tablets may be some hours after ingestion based on dissolution data from this study. For these tablets, users expecting a 'high' 15-30 minutes after ingestion may re-dose before peak concentrations for the first tablet have been reached, exacerbating any toxicity ${ }^{41}$. Healthcare workers should be aware that high-content, slowreleasing tablets are in circulation, and that users presenting with MDMA toxicity may not 
have reached peak plasma concentrations if they have ingested (multiple) slow-release tablets.

These data also impose an inherent limitation for rapid MDMA analysis. Whilst identification and content measurements can be made quickly and in remote locations, to assess dissolution profiles takes a much longer time (180 minutes using the method described, plus analysis time thereafter), and requires additional specialist equipment in a laboratory setting.

Knowledge of the MDMA content alone does not fully assess the risk for a tablet, or batch of tablets. The dissolution studies were conducted in standard aqueous buffer at acidic $\mathrm{pH}$ and whilst the findings of this study illustrate the potential variability in release rates from the tablets analysed, it should be noted that the in vitro - in vivo correlation of these data to predict physiological outcomes has not been confirmed. Further work would be needed in order to obtain this evidence. At present, there is no correlation with other tablet characteristics or parameters to identify fast- and slow-releasing tablets. In this work, we did not carry out any further tablet characterisation experiments (e.g. assessment of tablet hardness or friability) to correlate to dissolution, nor did we assess dissolution of any crushed tablets. Although the tablets were stored in dark, dry conditions and room temperature, we cannot be certain that storage did not have a detrimental effect on content. Finally, our data do not account for the inherent risk of illicitly manufactured drugs from non-pharmaceutical excipients, e.g. heavy metals, which should not be overlooked ${ }^{42}$. The nature of these and other diluents (often sugars such as lactose) needs to be explored.

\section{CONCLUSIONS}

Recreational MDMA use continues to be widespread. Recent concerns have focussed on the prevalence of high-content MDMA tablets in circulation, and our data from the UK support these observations.

However, in addition, we have demonstrated that variability in dissolution profiles of MDMA tablets must be considered. Rapid drug identification and quantitation are useful and transferable to non-laboratory settings, but in the light of these new data, do not and cannot fully-assess the risk to users due to the presence of fast- and slow-releasing MDMA tablets on the illicit market. 


\section{ACKNOWLEDGEMENTS}

The authors wish to thank Dr Christopher Benton and Dr lain Kay (Agilent Technologies) for provision of the Ultivo ${ }^{\mathrm{TM}}$ instrumentation used for the dissolution analyses in this study, Peter Cain (Eurofins Forensic Services Ltd.) for analysis of EQC samples, Dr Bob Boughtflower for helpful advice on pharmaceutical industry processes and release testing, and Hazan Hasan Sutcu (Queen Mary University of London) for assistance cataloguing MDMA tablets.

\section{REFERENCES}

1. Palamar JJ, Acosta P, Ompad DC, Cleland CM. Self-Reported Ecstasy/MDMA/"Molly" Use in a Sample of Nightclub and Dance Festival Attendees in New York City. Substance use \& misuse. 2017;52(1):82-91.

2. Roderick E, Penney J, Murrells T, Dargan PI, Norman IJ. Epidemiology of adolescent substance use in Norfolk schools. QJM : monthly journal of the Association of Physicians. 2018;111(10):699-706.

3. ONS. Office of National Statistics: Deaths related to drug poisoning in England and Wales: 2017 registrations. 2017; https://bit.ly/2OcAB3C. Accessed January 3rd, 2019.

4. Barratt MJ, Ferris JA, Winstock AR. Use of Silk Road, the online drug marketplace, in the United Kingdom, Australia and the United States. Addiction (Abingdon, England). 2014;109(5):774-783.

5. Aldridge J, Decary-Hetu D. Hidden wholesale: The drug diffusing capacity of online drug cryptomarkets. The International journal on drug policy. 2016;35:7-15.

6. Rhumorbarbe D, Staehli L, Broseus J, Rossy Q, Esseiva P. Buying drugs on a Darknet market: A better deal? Studying the online illicit drug market through the analysis of digital, physical and chemical data. Forensic science international. 2016;267:173-182.

7. Caudevilla F, Ventura M, Fornis I, et al. Results of an international drug testing service for cryptomarket users. The International journal on drug policy. 2016;35:38-41.

8. van der Gouwe D, Brunt TM, van Laar M, van der Pol P. Purity, adulteration and price of drugs bought on-line versus off-line in the Netherlands. Addiction (Abingdon, England). 2017;112(4):640-648.

9. EMCDDA. The European Monitoring Centre for Drugs and Drug Addiction: Recent changes in Europe's MDMA/ecstasy market - results from an EMCDDA trendspotter study. 2016; https://bit.ly/2LNkLMX. Accessed January 3rd, 2019.

10. ReagentTestsUK. Drug testing kits. 2019; https://www.reagent-tests.uk/. Accessed March 29th 2019.

11. EzTestKits. 2019; https://www.eztestkits.com/en/. Accessed March 29th 2019. 
12. Cole JC, Bailey M, Sumnall HR, Wagstaff GF, King LA. The content of ecstasy tablets: implications for the study of their long-term effects. Addiction (Abingdon, England). 2002;97(12):1531-1536.

13. Giraudon I, Bello PY. Monitoring ecstasy content in France: results from the National Surveillance System 1999-2004. Substance use \& misuse. 2007;42(10):1567-1578.

14. Shetab Boushehri SV, Tamimi M, Kebriaeezadeh A. Quantitative determination of 3,4-methylenedioxymethamphetamine by thin-layer chromatography in ecstasy illicit pills in Tehran. Toxicology mechanisms and methods. 2009;19(9):565-569.

15. Vogels N, Brunt TM, Rigter S, van Dijk P, Vervaeke H, Niesink RJ. Content of ecstasy in the Netherlands: 1993-2008. Addiction (Abingdon, England). 2009;104(12):2057-2066.

16. Khajeamiri AR, Kobarfard F, Ahmadkhaniha R, Mostashari G. Profiling of ecstasy tablets seized in iran. Iranian journal of pharmaceutical research : IJPR. 2011;10(2):211-220.

17. Morefield KM, Keane M, Felgate P, White JM, Irvine RJ. Pill content, dose and resulting plasma concentrations of 3,4-methylendioxymethamphetamine (MDMA) in recreational 'ecstasy' users. Addiction (Abingdon, England). 2011;106(7):1293-1300.

18. Togni LR, Lanaro R, Resende RR, Costa JL. The variability of ecstasy tablets composition in Brazil. Journal of forensic sciences. 2015;60(1):147-151.

19. Vidal Gine C, Ventura Vilamala M, Fornis Espinosa I, et al. Crystals and tablets in the Spanish ecstasy market 2000-2014: Are they the same or different in terms of purity and adulteration? Forensic science international. 2016;263:164-168.

20. Brunt TM, Nagy C, Bucheli A, et al. Drug testing in Europe: monitoring results of the Trans European Drug Information (TEDI) project. Drug testing and analysis. 2017;9(2):188-198.

21. Wood DM, Stribley V, Dargan PI, Davies S, Holt DW, Ramsey J. Variability in the 3,4-methylenedioxymethamphetamine content of 'ecstasy' tablets in the UK. Emergency medicine journal : EMJ. 2011;28(9):764-765.

22. USP. United States Pharmacopeia Convention Inc. 〈711〉 DISSOLUTION. 2016; https://bit.ly/2H5qfUL. Accessed January 3rd, 2019.

23. Anand O, Yu LX, Conner DP, Davit BM. Dissolution testing for generic drugs: an FDA perspective. The AAPS journal. 2011;13(3):328-335.

24. Szigeti B, Winstock AR, Erritzoe D, Maier L. Are ecstasy induced serotonergic alterations overestimated for the majority of users? Journal of psychopharmacology (Oxford, England). 2018;32(7):741-748.

25. TICTAC. Specialist drug identification, drug analysis and drug information. 2019; http://www.tictac.org.uk/. Accessed March 29th 2019.

26. Couchman L, Fisher DS, Subramaniam K, et al. Ultra-fast LC-MS/MS in therapeutic drug monitoring: Quantification of clozapine and norclozapine in human plasma. Drug testing and analysis. 2018;10(2):323-329. 
27. Sherlock K, Wolff K, Hay AW, Conner M. Analysis of illicit ecstasy tablets: implications for clinical management in the accident and emergency department. Journal of accident \& emergency medicine. 1999;16(3):194-197.

28. Asachi M, Nourafkan E, Hassanpour A. A review of current techniques for the evaluation of powder mixing. Advanced Powder Technology. 2018;29(7):15251549.

29. Spruit IP. Ecstasy use and policy responses in the Netherlands. Journal of Drug Issues. 1999;29(3):653-677.

30. Brunt T. Drug checking as a harm reduction tool for recreational drug users: opportunities and challenges; Background paper commissioned by the EMCDDA for Health and social responses to drug problems: a European guide. 2017; https://bit.ly/2zlUxNy. Accessed February 4th, 2019.

31. Tanner-Smith EE. Pharmacological content of tablets sold as "ecstasy": results from an online testing service. Drug and alcohol dependence. 2006;83(3):247-254.

32. Saleemi S, Pennybaker SJ, Wooldridge M, Johnson MW. Who is 'Molly'? MDMA adulterants by product name and the impact of harm-reduction services at raves. Journal of psychopharmacology (Oxford, England). 2017;31(8):1056-1060.

33. Vrolijk RQ, Brunt TM, Vreeker A, Niesink RJ. Is online information on ecstasy tablet content safe? Addiction (Abingdon, England). 2017;112(1):94-100.

34. Harper L, Powell J, Pijl EM. An overview of forensic drug testing methods and their suitability for harm reduction point-of-care services. Harm reduction journal. 2017;14(1):52.

35. Winstock AR, Wolff $K$, Ramsey J. Ecstasy pill testing: harm minimization gone too far? Addiction (Abingdon, England). 2001;96(8):1139-1148.

36. Murray DB, Potts $S$, Haxton $C$, et al. 'Ivory wave' toxicity in recreational drug users; integration of clinical and poisons information services to manage legal high poisoning. Clinical toxicology (Philadelphia, Pa). 2012;50(2):108-113.

37. Schneider J, Galettis P, Williams M, Lucas C, Martin JH. Pill testing at music festivals: can we do more harm? Internal medicine journal. 2016;46(11):12491251.

38. TICTAC. TICTAC spectral libraries for new psychoactive substances/legal highs. 2019; https://bit.ly/2Wtjkr2. Accessed March 29th 2019.

39. Dargan P, Wood D. Novel psychoactive substances: classification, pharmacology and toxicology. London: Academic Press; 2013.

40. NEPTUNE. Novel Psychoactive Treatment UK Network: Guidance on the Clinical Management of Acute and Chronic Harms of Club Drugs and Novel Psychoactive Substances. 2015; https://bit.ly/1HdQYm9. Accessed January 3rd, 2019.

41. Farre $M$, de la Torre $R$, Mathuna BO, et al. Repeated doses administration of MDMA in humans: pharmacological effects and pharmacokinetics. Psychopharmacology. 2004;173(3-4):364-375.

42. Varlibas F, Delipoyraz I, Yuksel G, Filiz G, Tireli H, Gecim NO. Neurotoxicity following chronic intravenous use of "Russian cocktail". Clinical toxicology (Philadelphia, Pa). 2009;47(2):157-160. 
